\title{
The relationship of isometric strength to peak dynamic hand forces during submaximal weight lifting
}

\author{
Deborah D. Thompson a , Don B. Chaffin a, Richard E. Hughes ${ }^{a}$ and Owen Evans ${ }^{b}$ \\ ${ }^{a}$ Center for Ergonomics, University of Michigan, Ann Arbor, MI 48109, USA \\ ${ }^{b}$ Department of Biosciences, LaTrobe University, Carlton, Victoria Australia
}

(Received February 6, 1991; accepted in revised form August 3, 1991)

\begin{abstract}
Reports have indicated that approximately one third of the US workforce is presently required to exert significant strength as part of their jobs. In addressing the prevention of these types of injuries, the assessment of muscular strength can provide a method of predicting whether a person is capable of performing the job without incurring injury. Currently, isometric strength assessments are often used to assist in predicting the capability to safely perform a lifting task. However, given the dynamic nature of work activities, isometric values may be limited, particularly if the load is well below a person's strength capability. Thus, it is of interest to evaluate the relationship between isometric strength values and peak dynamic hand forces under varied submaximal loading. It is shown in this study that the peak hand forces, exhibited while dynamically lifting different submaximal loads, are not highly correlated with a person's isometric lifting strength in similar postures. It is also shown that for very light load lifting, the peak accelerations approached $2.5 \mathrm{~g}$ 's, further supporting the need to limit such lifting from floor level by using ergonomic interventions.
\end{abstract}

\section{Relevance to industry}

Back and overexertion injuries are a major concern in industry today. This paper examined the behavior of peak hand forces during dynamic lifts of submaximal loads (which are common to industry), and whether they could be predicted by isometric strength tests. If a statistical relationship exists between isometric strength and submaximal dynamic lifting performance, industry could continue to use the available isometric strength testing methods to screen workers for jobs with submaximal load lifting requirements. The paper questions whether this will be possible, in the future, for light loads.

\section{Keywords}

Isoinertial strength, lifting, isometric strength.

\section{Introduction}

\section{Background}

It has been well documented that back and overexertion injuries are a major problem (in terms of injury and economic costs) in industry today. A NIOSH (National Institute for Occupational Safety and Health) report (NIOSH, 1981) states that approximately one third of the US workforce is presently required to exert signifi- cant strength as part of their jobs. It also states that overexertion injuries account for about one fourth of all reported occupational injuries in the US. Statistics from the State of Michigan (Michigan Department of Labor, 1986) also confirm these numbers since in 1985 , overexertion was the most frequently reported cause of injury $(34.3 \%)$. So the issue is no longer whether or not the problem exists, the concern now is the prevention of these types of injuries. In addressing this concern, NIOSH (1981) recommends two ap- 
proaches, namely engineering and administrative controls, in the prevention of back and overexertion injuries.

The goal of engineering control methods is to engineer the hazard out of the job, namely, eliminate the most stressful tasks (manual material handling activities, in most cases) and/or modify workplace designs which lead to overexertion injuries. However, there is some speculation that there exists a prevailing philosophy in industry, that the costs associated with engineering controls (redesign, automation, equipment, etc.) are excessive and cannot always be justified. Also, some needed changes are technically infeasible. Therefore, many have focused their attention on instituting administrative controls.

One type of administrative control method is the selection or screening of workers (in order to match individual capabilities with the job demands) by way of strength assessments. This has been one area that has received a fair amount of attention. The assessment of muscular strength can provide a method of predicting whether an individual is capable of performing the job without incurring injury. Since muscular strength is most often required on jobs involving manual material handling, if an individual's strength is not sufficient to meet the demands of the job, then exertion related injuries are more likely to occur. However, the screening of workers involves complicated social, economic, moral and legal issues which affect the usability of many selection procedures. For example, current Federal legislation concerning equal employment opportunities sets clear guidelines regarding the use of specific testing procedures. To be considered legal and non-discriminatory, testing procedures must clearly be job related and must actually make predictions of the individual's ability to safely perform the necessary tasks within the job in question. So a major problem which exists with current techniques of strength testing is that of validity, as it relates to the ability of the tests to predict specific job performance.

\section{Isometric vs. dynamic strength testing}

Studies of others have indicated that workers who were placed in jobs where the strength requirements exceeded their job specific isometric strength capabilities had incidents of strains and sprains that were three times higher than those whose isometric strength exceeded the job strength requirements (Chaffin et al., 1976). Thus, it is generally accepted that the assessment of job specific isometric strength provides a method of predicting whether a worker is capable of performing a lifting task without incurring injury or strain. This raises the issue of predicting the future risk of injury as opposed to predicting job performance capability (Garg et al., 1980).

However, manual material handling activities are generally dynamic in nature, and isometric strength testing only evaluates strength in one position devoid of joint movement. It does not allow for dynamic changes in posture, joint loading and muscle velocity-tension relationships, nor does it account for acceleration and deceleration of body segments. Therefore, the isometric strength values may be limited.

Given the dynamics of work activities, dynamic strength testing has been proposed to be more predictive of job strength performance, but such dynamic testing is more complex, due to the number of parameters (velocity, acceleration, jerk, etc.) that must be controlled. Also, the validity of dynamic strength testing has been based on a psychophysical method, wherein the subjects are allowed to adjust the load lifted until they obtain, in their judgement, the maximum amount of weight that can be lifted safely (Snook, 1978). This maximal acceptable load is then compared to their strength test results (Aghazadeh and Ayoub, 1985). In reality, a worker normally does not adjust the weight of a load. Usually, constant loads are presented, and the worker is required to lift the load, by whatever means necessary, in order to perform the task. Therefore, the exertion is conducted over the range of motion with a constant mass (isoinertial method).

\section{Why submaximal loads?}

It is understood that heavy/maximal load lifting contributes to increasing the incidence and severity of musculoskeletal injuries, while lifting light/submaximal loads results in fewer incidents and a reduced severity in the injuries which do occur (NIOSH, 1981). However, the fact remains that injuries do occur, and if submaximal loads 
are lifted quickly, the dynamic forces imparted by the rapid motion can multiply a load's effect. Research has suggested that when large acceleration or jerk forces occur, the stress on the musculoskeletal system can result in the generation of muscular force and spinal loading levels which exceeds one's maximum level of tolerance, thereby increasing the risk of injury (Ayoub and El-Bassousi, 1978; Leskinen et al., 1983; Marras et al., 1987). Therefore, the lifting of submaximal loads should be of concern when assessing conditions which can result in back and overexertion injuries.

\section{Objective}

The objectives of this study were twofold. First, it was necessary to determine how the peak hand forces varied during lifts of submaximal loads when performed by people of varied strengths. This information could then be used to determine if peak hand forces, during dynamic lifts with set submaximal loads, could be predicted by isometric strength tests.

Isometric strength testing has been shown to meet general test criteria for the physical assessment of workers for manual material handling
Table 1

Summary of age, body weight and stature of subjects.

\begin{tabular}{lrcr}
\hline Variable & Mean & Std. dev. & \multicolumn{1}{l}{ Range } \\
\hline Age (yrs) & 25.8 & 4.2 & $21.0-31.0$ \\
Body weight (kg) & 76.1 & 12.4 & $66.2-110.2$ \\
Stature (cm) & 178.8 & 8.7 & $170.2-200.7$ \\
\hline
\end{tabular}

tasks (Chaffin, 1982). So from an economic and validation standpoint, if a statistical relationship exists between isometric strength and submaximal dynamic lifting performance, industry could continue to use the available isometric strength testing methods to screen workers for jobs with submaximal load lifting requirements. Otherwise, the complex task of developing and validating dynamic strength testing techniques will have to be pursued.

In this study, an attempt was made to simulate submaximal weight lifting, which is common to industry, while carefully measuring the peak dynamic (isoinertial) hand forces and postures displayed by the subjects (whose isometric lifting strengths were obtained in a variety of postures similar to those used in the different phases of the dynamic lifts). (a)

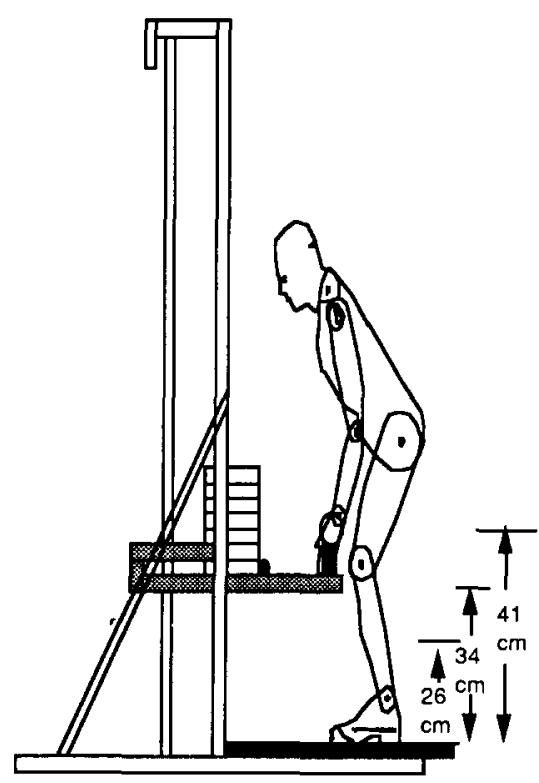

(b)

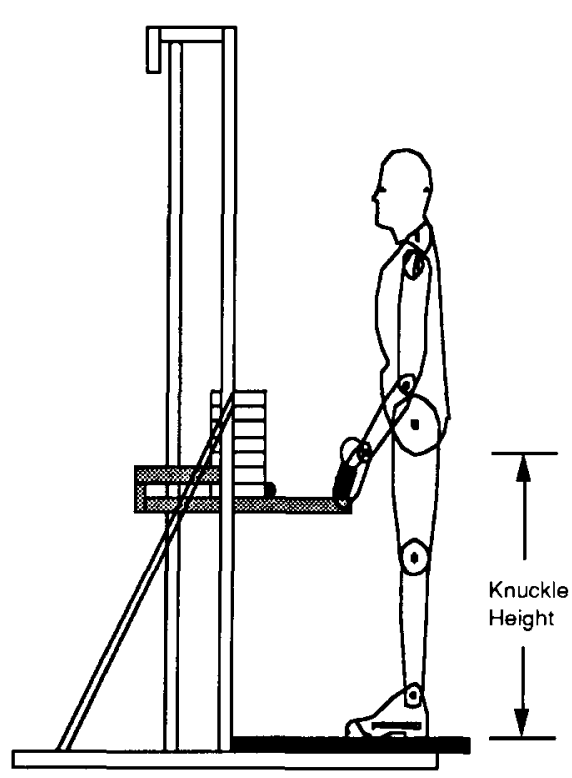

Fig. 1. Strength testing fixture for (a) isometric and (b) dynamic (isoinertial) lifting assessments. 


\section{Method}

\section{Subjects}

Ten male subjects volunteered to participate in the study. They were of good health, with no prior history of back pain nor injury. Their ages, body weight and statures are provided in table 1.

The subjects were informed of the purpose of the study, and were familiarized with the experimental procedures. Each subject read and signed a consent form prior to their participation. They were paid on an hourly basis.

\section{Apparatus}

The subjects were required to exert a lifting force on a strength testing fixture, the Force Evaluation and Testing System, manufactured by Hoggan Health Industries of Salt Lake City, UT. The test fixture allowed for the two-handed, sagittal plane lifting assessment of isometric and dynamic (isoinertial) lifting strength (figure 1). It also allowed for an unrestricted lifting posture.

The arm assembly of the unit was equipped with a handle which was connected to a load cell. The arm was secured so that the handle would not translate toward or away from the subject during the lifting activity. Also, the load cell was hinged to the arm so that the handle was allowed to rotate in order to simulate normal lifting conditions. Usually, when a load is lifted, it is both vertically lifted and pulled in toward the body (Garg et al., 1980). Therefore, allowing the handle to rotate resulted in a better simulation of the actual load lifting trajectory.

A force monitor amplified the signal received from the load cell for input into a 12-bit, analogto-digital converter. A potentiometer, in series with a battery, was attached to the test fixture's pulley in order to provide handle position data. Both hand force and handle position were sampled at $30 \mathrm{~Hz}$ and stored on a Zenith 386 microcomputer.

\section{Procedure}

The subjects were allowed to perform practice trials for both the isometric and dynamic test conditions. The purpose of the practice trials was to familiarize the subject with the testing fixture, and to allow them to determine their own lifting posture (Stevenson et al., 1990b). Allowing the subjects to freely choose a lifting posture was based on research presented in a NIOSH report (NIOSH, 1981) that indicated that there is not one 'correct' lifting posture. The report also mentions that it may be safer to allow workers to use their own common sense and to monitor their own feeling of the exertion in choosing an appropriate lifting posture. Once they chose their lifting posture, the foot position was recorded and it was required that the subject assume the same basic posture, as established by the foot position, for all subsequent lifting trials.

For each subject, the strength measurements were the average isometric and peak dynamic hand forces exerted on the handle. Peak dynamic hand force needed to perform the lifting task was the recorded measure since the interest of the study was in the force needed to lift an object (lifting force) as opposed to a sustained force, which would be needed in carrying and holding type activities.

\section{Isometric strength measurements}

The isometric strength testing was performed according to procedures recommended by Caldwell et al. (1974) and Chaffin (1975). Trials were conducted with the handle located at three different heights (hand locations of 26, 34 and 41 centimeters as measured vertically from the platform upon which the subjects stood - see figure 1). Three trials were conducted at each of the heights, and the order sequence of the trials were randomized.

Before each measurement, the subjects were instructed to lift up on the handle as hard as possible, without jerking, and maintain or 'hold' the exertion until told to stop. The trials were designed such that force data was sampled for a duration of 3 seconds during the maximum exertion phase of the trial, with a 2-minute rest period between trials in order to reduce the possibility of fatigue (Caldwell et al., 1974, and Chaffin, 1975). The hand forces exerted during the 3 -second exertion were recorded. No verbal encouragement, nor performance feedback was provided. 


\section{Dynamic (isoinertial) strength measurements}

The isometric tests were performed first in order to determine the subject's lifting capacity (maximum value). Submaximal loads, varying from $7 \mathrm{~kg}$ to $48 \mathrm{~kg}$, were chosen to simulate lifting tasks which were common to industry. The upper load range lifted for each subject varied however, in order to avoid overexertion risks for weaker subjects. The maximal load lifted was set at $75 \%$ of isometric lift strength. Consequently, the heaviest load lifted was set at either $34 \mathrm{~kg}$ or $48 \mathrm{~kg}$. For each of the loads lifted, three trials were performed, with the order sequence of the trials randomized.

Before each measurement, the subjects were instructed to lift the load, from the initial height of $26 \mathrm{~cm}$ (as measured vertically from the platform of the testing fixture) to their standing knuckle height (see figure 1). This facilitated smooth lifting since no readjustments in the hand position were necessary to lift higher than knuckle height (Stevenson et al., 1990a). They were instructed to lift smoothly, at a comfortable speed, as if they would lift an object under actual conditions. They were informed to maintain or 'hold' the load at knuckle height until told to stop. The trials were designed such that data was sampled for a duration of 3 seconds during the lift, with a 2 -minute rest period between trials. No verbal encouragement, nor performance feedback was provided.
Table 2

Pooling of mean isometric strength to develop isometric lifting strengths for each subject.

\begin{tabular}{lc}
\hline Subject & $\begin{array}{l}\text { Isometric lifting } \\
\text { strenth }(\mathrm{N})\end{array}$ \\
\hline 1 & 546 \\
2 & 700 \\
3 & 680 \\
4 & 818 \\
5 & 652 \\
6 & 1202 \\
7 & 742 \\
8 & 931 \\
9 & 1104 \\
10 & 439 \\
\hline
\end{tabular}

\section{Results and discussion}

\section{Isometric strength measurements}

The results of the trials, conducted at the hand locations of 26, 34 and 41 centimeters, are summarized in figure 2 . For each subject, at each hand location height, the hand force measurements for each of the three trials were averaged, resulting in the mean isometric strength plotted in figure 2 .

In reviewing figure 2 , given the similarities in the graph from one height to another, the first question that arises is whether there is a significant change in the mean isometric strength as one moves from one height to another, i.e., does the height have an affect on strength? An analy-

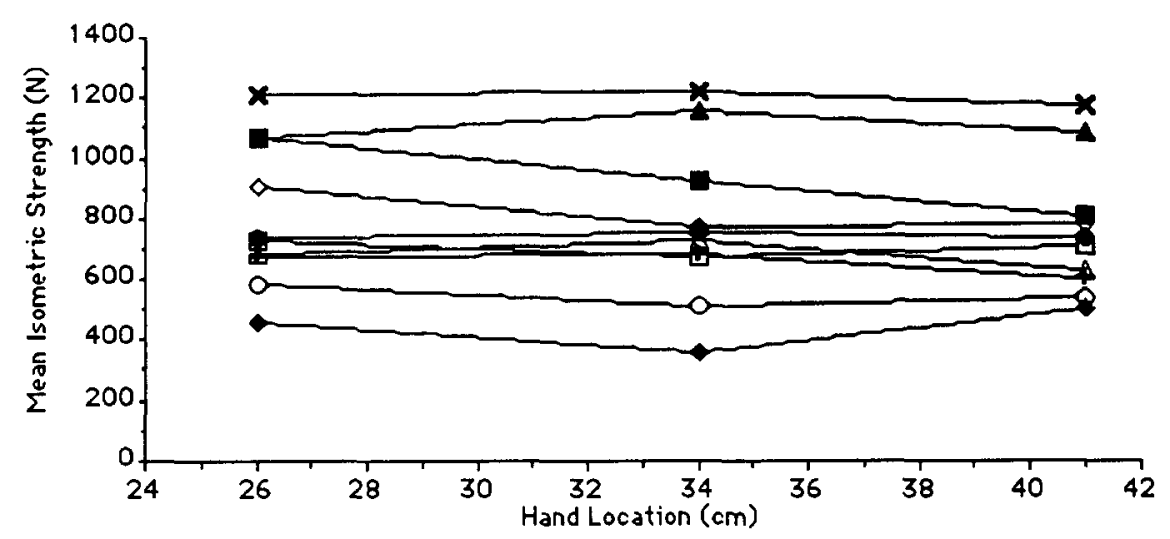

Fig. 2. Graphical representation of the mean isometric strength for the ten subjects at each hand location height. 
sis of variance was conducted to determine if the strength measures are dependent on the hand location height, and the results revealed that the mean isometric strength measurements could not be shown to be significantly different at the different hand location heights $(F(2,27)=0.13, p>$ $0.05)$. Therefore, it was deemed appropriate to pool the mean isometric strength measurements to develop an isometric lifting strength measurement for each subject (table 2), which is the measure that will be used as the basis for comparison with peak dynamic (isoinertial) hand forces.

Dynamic (isoinertial) submaximal strength measurements

The results of the dynamic trials which were conducted with submaximal lifting loads ranging from $7 \mathrm{~kg}$ to $48 \mathrm{~kg}$ (67 to 467 Newtons), are summarized in figure 3 . For each subject at each of the lifting loads, the peak hand force measurement for each of the three trials were averaged, which resulted in the peak dynamic hand forces graphed in figure 3.

The graphical results and the $r^{2}$ value of 0.96 indicate a highly linear relationship between the load lifted and the peak dynamic hand forces, i.e., the hand forces increased significantly with increased load lifted. Given this relationship, it was deemed appropriate to identify the peak dynamic hand forces which will form the basis of the comparison later with isometric lifting strengths of each subject. The maximum peak
Table 3

Maximum peak dynamic hand forces for each of the ten subjects.

\begin{tabular}{ll}
\hline Subject & $\begin{array}{l}\text { Maximum peak dynamic } \\
\text { hand force }(\mathrm{N})\end{array}$ \\
\hline 1 & 547 \\
2 & 760 \\
3 & 540 \\
4 & 544 \\
5 & 524 \\
6 & 756 \\
7 & 544 \\
8 & 794 \\
9 & 783 \\
10 & 508 \\
\hline
\end{tabular}

dynamic hand force for each subject (table 3), which is the hand force exerted when lifting the heaviest load a specific subject was allowed to lift (either $34 \mathrm{~kg}$ or $48 \mathrm{~kg}$ ), is the measure that will be used as the basis for this comparison.

Further analysis of the graphical results reveals that the regression line does not pass through the origin. This is due, in part, to the fact that during the lifting task, not only is the load lifted, the subject must also lift their upper body. Thus, the intercept represents the effect due to lifting one's body mass. Also in figure 3, a line of direct proportion was included to indicate when the load lifted equals the peak hand forces $(F=$ $\mathrm{mg}$ ) without additional inertial effects due to motion. This would occur with very slow lifts

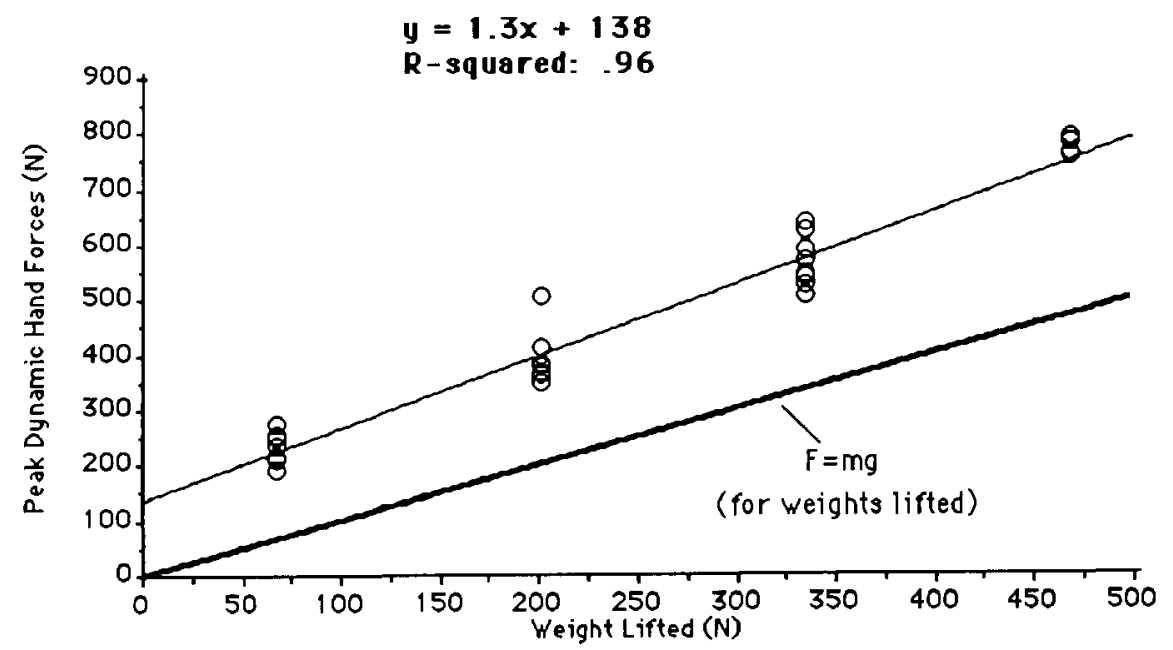

Fig. 3. Graphical representation of the peak dynamic hand forces for the ten subjects at each weight lifted. 


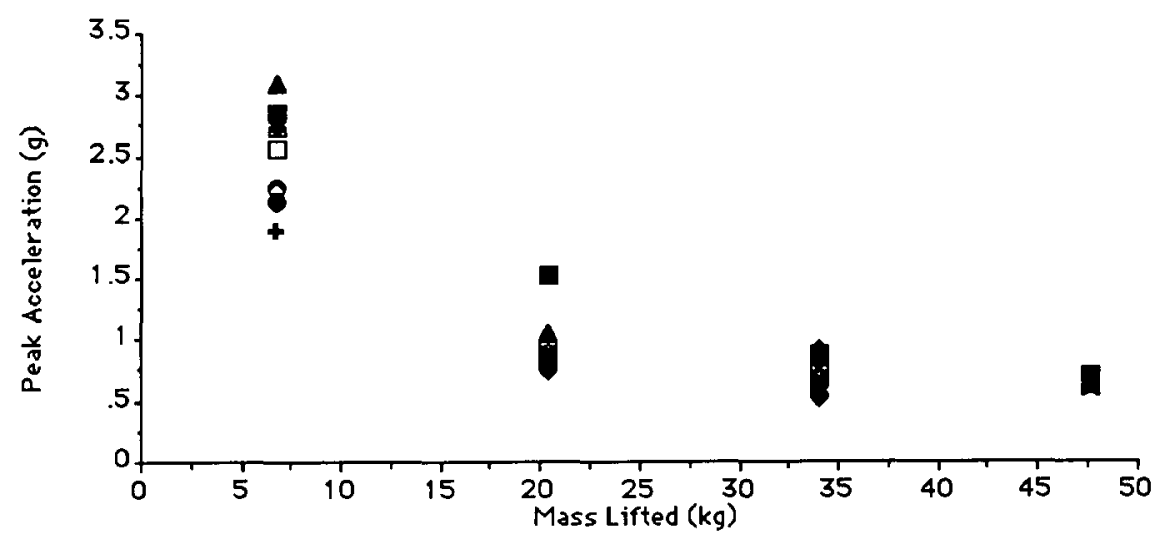

Fig. 4. Peak accelerations demonstrated by each subject during the lifting of the various submaximal loads.

(negligible acceleration) where no inertial effect is in operation. Since the resulting regression line was found to be above the line of direct proportion, it indicates that a positive inertial effect ( $F=\mathrm{mg}+\mathrm{ma}$ ) is occurring, and this effect tends to increase as the load lifted was increased. Therefore, the forces imparted to the subjects depended both on the mass being lifted, and the accelerations imparted to it and their body segments during the lift. Given this result, it was necessary to analyze the acceleration effects with the lifting of submaximal loads in order to understand the inertial effect. A plot of the accelerations is shown in figure 4 . The acceleration values were derived by using the inertial effect deriva- tion $(F=\mathrm{mg}+\mathrm{ma})$, and solving for the acceleration.

Although there was a positive inertial effect for all loads (figure 3), the accelerations decreased with the increasing loads (figure 4). Research by Carlsöö (1980) indicates that the increased inertial effect is due to an increase in the mass, which more than offsets the decreased acceleration with the heavier loads. The results here are consistent with Carlsöö's research. Interestingly enough, at the heavier submaximal loads (34 and $48 \mathrm{kgs}$ ) the subjects demonstrated a relatively constant peak acceleration $(0.5-0.7 \mathrm{~g})$ when handling loads between $40 \%$ and $75 \%$ of their isometric strength values. This result supports the

\section{Table 4}

Comparison of isometric and the maximum values of the peak dynamic force measurements for each subject.

\begin{tabular}{llll}
\hline Subject & $\begin{array}{l}\text { Isometric lifting } \\
\text { strength }(\mathrm{N})\end{array}$ & $\begin{array}{l}\text { Maximum peak dynamic } \\
\text { hand forces }(\mathrm{N})\end{array}$ & $\begin{array}{l}\text { Ratio of dynamic to } \\
\text { isometric strengths }\end{array}$ \\
\hline 1 & 546 & 547 & 1.00 \\
2 & 700 & 760 & 1.08 \\
3 & 680 & 540 & 0.79 \\
4 & 818 & 544 & 0.67 \\
5 & 652 & 524 & 0.80 \\
6 & 1202 & 756 & 0.63 \\
7 & 742 & 544 & 0.73 \\
8 & 931 & 794 & 0.85 \\
9 & 1104 & 783 & 0.71 \\
10 & 439 & 508 & 1.16 \\
Mean & 781 & 630 & \\
\hline
\end{tabular}


findings of Leskinen (1985) who recorded peak accelerations of $0.49-0.6 \mathrm{~g}$ for males lifting a 15 $\mathrm{kg}$ box to knuckle height.

\section{Isometric us. dynamic}

The isometric lifting strengths and maximum peak dynamic hand force measurements (in Newtons) for each subject are summarized in table 4 . Although the maximum peak dynamic hand forces were based on lifting submaximal loads for most of the trials, it appears that for some subjects $(1$, 2 and 10), these loads represented maximal exertions, since their maximum peak dynamic hand forces ranged from $100 \%$ to $116 \%$ of their isometric lifting strengths.

A comparison was made between the ratio of the maximum peak dynamic hand forces and the isometric lifting strength for the group of subjects versus their individual strength ratios (table 4). The group comparison yielded a ratio of 0.80 , while there was variation in the individual comparisons, ranging from 0.63 to 1.16 . Therefore, for a given subject, peak hand forces under heavy submaximal loading conditions cannot be predicted with accuracy from the isometric strengths. This result is consistent with the results reached by other researchers (Garg et al., 1982; Kroemer, 1983), which were conducted with maximal dynamic loads.
A regression analysis was conducted to determine if any relationship exists between isometric lifting strengths and the maximum peak dynamic hand forces displayed by each subject lifting the submaximal loads (figure 5). The results indicate a moderate correlation $(r=0.75)$. However, the regression model only explains $57 \%$ of the observed variation in determining one's dynamic hand force given a known isometric lifting strength. Since $43 \%$ of the variation in the hand forces remains unexplained by the model, an empirical relationship cannot be proposed between isometric lifting strength and the maximum values of peak dynamic hand forces for the submaximal loads set for these subjects.

Inspection of the graph in figure 5 clearly indicates that the subjects chose rather constant peak hand forces during the two heavier submaximal load lifting conditions ( 34 or $48 \mathrm{kgs}$ ), regardless of their isometric lifting strengths. The basis for the peak forces probably depends more on speed of muscle contraction and coordination than isometric muscle strength. Also, peak dynamic hand force measurements exhibited a more consistent performance amongst the subjects (coefficient of variation $=3.69 \%$ ) as compared to the isometric strength measurements (coefficient of variation $=7.94 \%$ ). Therefore, it would appear that dynamic measurements of one's lifting capability will be less variable in its prediction than that of isometric strength tests.

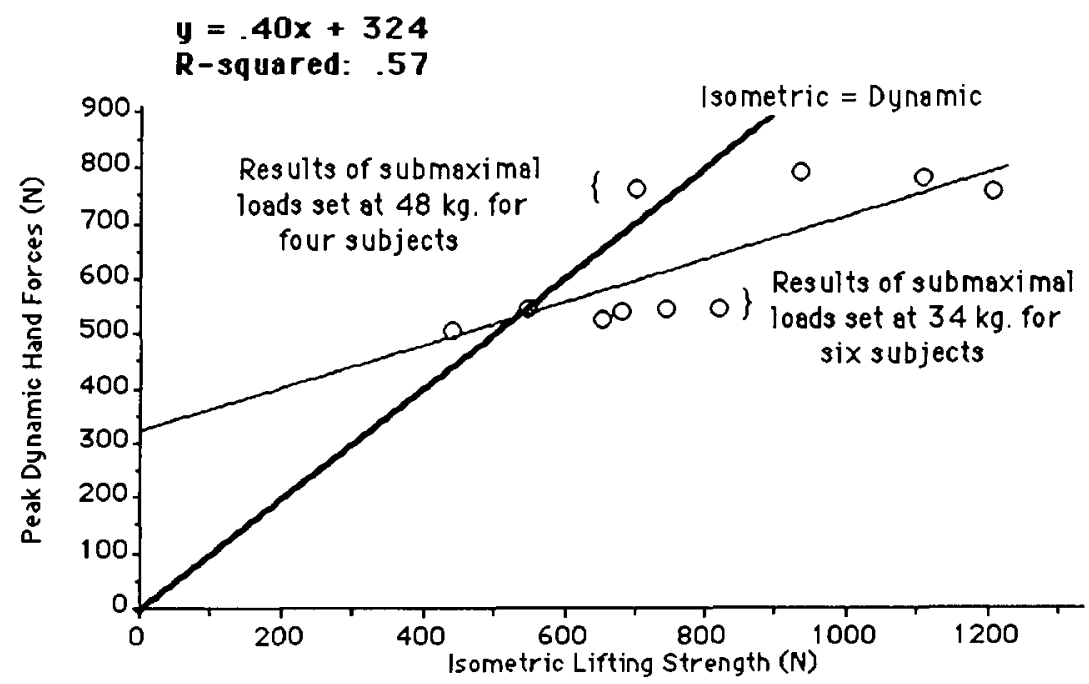

Fig. 5. Regression analysis of isometric lifting strength versus the maximum values of the peak dynamic hand forces. 


\section{Conclusion}

In comparing the hand forces for isometric and submaximal isoinertial lifting, a regression analysis of the maximum dynamic peak values determined that they were not well correlated to isometric lifting strength values. One's peak dynamic hand force, when lifting submaximal loads, cannot be predicted, with any reasonable accuracy, from isometric strength tests. Further research is needed in the area of dynamic strength testing to determine if such tests will better predict peak hand forces during submaximal lifting.

Although this research addresses the question of whether peak hand forces during submaximal load handling can be predicted by isometric strength testing, it also raises additional questions, which will require further review. For example:

(1) Would the same results be reached for female subjects?

(2) Would the same results be reached if the hand forces were measured under maximal loading conditions?

(3) Why did some subjects, who exhibited high isometric values, not exhibit high dynamic values (i.e., see figure 5 for those subjects whose values were below the line of direct proportion used to indicate where isometric values equal dynamic values)?

(4) Under heavier loading conditions (i.e., above $75 \%$ of isometric strength), will subjects continue to lift with a relatively constant acceleration of about $0.5-0.7 \mathrm{~g}$ ?

Addressing these questions could form the basis for future research activities.

\section{Acknowledgements}

The authors wish to acknowledge the support of The Association of American Railroads (contract SFR-87-024), Rush-Presbyterian-St. Luke's Medical Center (NIH Grant RO1AR39599), and The Center for Ergonomics Affiliates Program.

\section{References}

Aghazadeh, F. and Ayoub, M.M., 1985. A comparison of dynamic- and static-strength models for prediction of lifting capacity. Ergonomics, 28: 1409-1417.
Ayoub, M. and El-Bassousi, M., 1978. Dynamic biomechanical model for sagittal plane lifting activities. Safety in Manual Materials Handling. NIOSH, Cincinnati, $\mathrm{OH}$.

Caldwell, L.S., Chaffin, D.B., Dukes-Dobos, F.N., Kroemer, K.H.E., Laubach, L.L., Snook, S.H. and Wasserman, D.E., 1974. A proposed standard procedure for static muscle strength testing. AIHA Journal, 35: 201-205.

Carlsöö, S., 1980. A back and lift test. Applied Ergonomics, 11: $66-72$.

Chaffin, D.B., 1975. Ergonomics guides - Ergonomics guide for the assessment of human static strength. AIHA Journal, 36: 505-511.

Chaffin, D.B., 1982. Functional assessment for heavy physical labor. Clinical Medicine for the Occupational Physician. Marcel Dekker, Inc., New York, pp. 87-192.

Chaffin, D.B., Herrin, G.D., Keyserling, W.M. and Foulke, J.A., 1976. Preemployment strength testing of selected workers for material handling jobs. NIOSH, Cincinnati, $\mathrm{OH}$.

Garg, A., Chaffin, D.B. and Freivalds, A., 1982. Biomechanical stresses from manual load lifting: A static vs. dynamic evaluation. IIE Transactions, 14: 272-280.

Garg, A., Mital, A. and Asfour, S.S., 1980. A comparison of isometric strength and dynamic lifting capability. Ergonomics, 23: 13-27.

Kroemer, K.H.E., 1983. An isoinertial technique to assess individual lifting capability. Human Factors, 25: 493-506.

Leskinen, T.P.J., 1985. Comparison of static and dynamic biomechanical models. Ergonomics, 28: 285-291.

Leskinen, T.P.J., Stalhammar, H.R., Kuorinka, I.A.A. and Troup, J.D.G., 1983a. The effect of inertial factors on spinal stress when lifting. Engineering in Medicine, 12: 87-89.

Leskinen, T.P.J., Stalhammar, H.R., Kuorinka, I.A.A. and Troup, J.D.G., 1983b. A dynamic analysis of spinal compression with different lifting techniques. Ergonomics, 26 : 595-604.

Marras, W.S., Rangarajulu, S.L. and Lavender, S.A., 1987. Trunk loading and expectations. Ergonomics, 30: 551-562.

Michigan Department of Labor, 1986. Compensable occupational injury and illness report, Michigan 1985. Bureau of Safety \& Regulation.

NIOSH, 1981. Work practices guide for manual lifting. NIOSH, Cincinnati, $\mathrm{OH}$.

Snook, SH., 1978. The design of manual handling tasks. Ergonomics, 21: 963-985.

Stevenson, J.M., Bryant, J.T., French, S.L., Greenhorn, D.R., Andrew, G.M. and Thomson, J.M., 1990a. Dynamic analysis of isoinertial lifting technique. Ergonomics, 33: 161-172.

Stevenson, J., Bryant, J., Greenhorn, D., Smith, T., Deakin, J. and Surgenor, B., 1990b. The effect of lifting protocol on comparisons with isoinertial lifting performance. Ergonomics, 33: 1455-1469. 